\title{
Modelagem Matemática de Secagem de Grãos: Aplicação do Método dos Volumes Finitos
}

P.A.P. BORGES 1 , Departamento de Física, Estatística e Matemática, UNIJUÍ e PROMEC-UFRGS, Rua São Francisco, 501 Cx.P. 560 Ijuí, RS, Brasil

O.A. KHATChatOURIAN ${ }^{2}$, Departamento de Física, Estatística e Matemática, UNIJUÍ, Ijuí, RS, Brasil

H.A. VIELMO ${ }^{3}$, Departamento de Engenharia Mecânica, UFRGS, RS, Brasil.

\section{Introdução}

A pesquisa de modelos matemáticos para secagem de grãos, nas últimas décadas, teve grande avanço e combinada com os métodos experimentais, vem tornando-se uma alternativa eficiente de geração de informações técnicas para projeto, construção e monitoramento de unidades de secagem.

Nos modelos de camada fina, onde o objetivo é descrever como se dá o transporte de umidade dentro do grão, predominam os fenômenos de difusão. Nos modelos para leito profundo, além daquele objetivo, interessam também os fenômenos de transporte da umidade nos espaços entre os grãos, que é um fenômeno predominantemente convectivo. Vários modelos de secagem para leito profundo foram propostos, tais como os modelos logarítmicos e os que envolvem sistemas de equações diferenciais parciais (EDP). O modelo de Brooker et al. [2] é constituído por quatro EDPs,

pborges@unijui.tche.br

olegkha@unijui.tche.br

vielmoh@mecanica.ufrgs.br 
e serviu de base para outros modelos. Courtois et al. [3] modelaram a secagem de milho. França [4] considerou um secador bi-dimensional e resolveu o sistema utilizando os métodos de elementos finitos e volumes finitos.

Com o objetivo de fazer um software para modelagem da secagem de soja, Khatchatourian et al. [5] criaram um modelo matemático com um sistema de quatro equações diferenciais parciais, utilizando parâmetros físicos conhecidos e cálculo dos fluxos de massa e calor, a partir de dados experimentais. A necessidade de uma execução computacional rápida levou à pesquisa de métodos numéricos adequados para esse fim. Resolveram o sistema usando diferenças finitas.

Este trabalho dá continuidade à pesquisa de métodos numéricos para a resolução do sistema referido acima, implementando o método dos volumes finitos. Os resultados foram comparados com o método das diferenças finitas. Foi feita a análise de independência de malha, assim como implementados diferentes esquemas de interpolação.

\section{Modelo Físico}

O secador modelado neste trabalho é do tipo leito fixo, ilustrado na Fig.1. É constituído por uma caixa completamente cheia de grãos, nesse caso soja, pela qual passa um fluxo de ar ambiente aquecido. O aumento da temperatura faz com que aumente a capacidade do ar para absorver umidade. Ao passar pela camada de grãos úmidos, o ar seco reduz o teor de umidade de equilíbrio, provocando um fluxo de vapor de água, do grão para o ar. Esta água é transportada pelo ar para fora do secador. Como a largura do leito é muito maior do que o comprimento das camadas limite de velocidade e temperatura, o modelo foi considerado unidimensional e a velocidade do fluxo de ar constante.

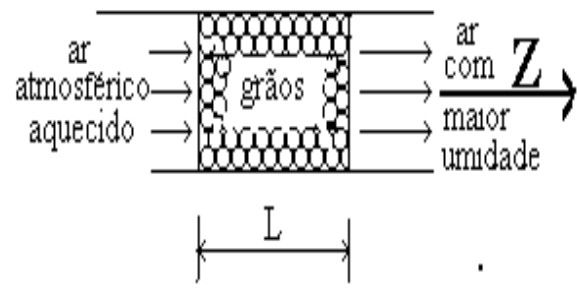

Figura 1: Secador unidimensional de leito fixo

\section{Modelo Matemático}

Em Katchatourian et al. [5] encontra-se a dedução das equações do modelo matemático para secagem de soja, baseado em dados empíricos dos fluxos de massa $\left(\Phi_{m}\right)$ e calor $\left(\Phi_{h}\right)$. 


$$
\begin{aligned}
\frac{\partial X}{\partial t} & =-\frac{\Phi_{m} a}{\rho_{g}}, \\
\frac{\partial T_{g}}{\partial t} & =\frac{-\Phi_{h} a-\Phi_{m} a L_{v}}{\rho_{g}\left(C_{p g}+X C_{p w}\right)}, \\
\frac{\partial Y}{\partial t} & =-V_{a} \frac{\partial Y}{\partial Z}+\frac{a \Phi_{m}(1-\epsilon)}{\rho_{a} \epsilon}, \\
\frac{\partial T_{a}}{\partial t} & =-V_{a} \frac{\partial T_{a}}{\partial Z}+\frac{a(1-\epsilon)}{\rho_{a} \epsilon} \frac{\Phi_{m} C_{p v}\left(T_{g}-T_{a}\right)+\Phi_{h}}{C_{p a}+C_{p v} Y},
\end{aligned}
$$

onde $\mathrm{X}$ e Y são os teores de umidade e $T_{g}$ e $T_{a}$ são as temperaturas do grão e do ar respectivamente.

As condições iniciais são: $\mathrm{X}=\mathrm{X} 0 ; T_{g}=T_{g} 0 ; \mathrm{Y}=\mathrm{Y} 0$ e $T_{a}=T_{a} 0$ em $(0, \mathrm{z})$ para $0 \leq z \leq L \mathrm{e}$ as condições de fronteira são $\mathrm{Y}=\mathrm{Y}(\mathrm{t}, 0)$ e $\mathrm{Ta}=T_{a}(\mathrm{t}, 0)$ para $0 \leq t<\infty$.

Os fluxos de massa e calor são termos variáveis no tempo e no espaço e dependem das quatro variáveis principais, determinando um forte acoplamento entre as equações.

A convergência da solução numérica do sistema (3.1)-(3.4), usando o esquema explícito com passos espaciais razoáveis ( 9 a 121 volumes), só é possível com um passo temporal muito pequeno, da ordem de 0,0001s. Para tempos de secagem maiores do que $1 \mathrm{~h}$ essa escolha torna-se inviável. Courtouis et al. [3], com a intenção de criar um modelo simplificado, que usasse menos tempo computacional, negligenciaram as taxas de variação da umidade e temperatura do ar em relação ao tempo, em comparação com as taxas de variação dessas variáveis em relação ao espaço. Khatchatourian et al. [6], mostraram que as ordens de grandeza daquelas taxas de variação são realmente muito diferentes e que os resultados obtidos neglicenciando as derivadas temporais, são praticamente iguais. Esse modelo simplificado será chamado neste trabalho de pseudo-implícito. Assim, as equações (3.3)-(3.4) ficaram com a seguinte forma:

$$
\begin{aligned}
V_{a} \frac{\partial Y}{\partial Z} & =\frac{a \Phi_{m}(1-\epsilon)}{\rho_{a} \epsilon} \\
V_{a} \frac{\partial T_{a}}{\partial z} & =\frac{a(1-\epsilon)}{\rho_{a} \epsilon} \frac{\Phi_{m} C_{p v}\left(T_{g}-T_{a}\right)+\Phi_{h}}{C_{p a}+C_{p v} Y} .
\end{aligned}
$$

\section{Método de Solução}

A maioria dos trabalhos relacionados com simulação do processo de secagem de grãos usa o método de diferenças finitas para resolver o sistema de EDPs. Neste trabalho, o sistema de equações (3.1), (3.2), (3.5) e (3.6) foi resolvido usando o método dos volumes finitos. Cada equação foi integrada e expressa na forma discreta, mediante as seguintes considerações:

1. O domínio de integração foi dividido em células unidimensionais de comprimento $\Delta z$, como indica a Fig. 2 . 


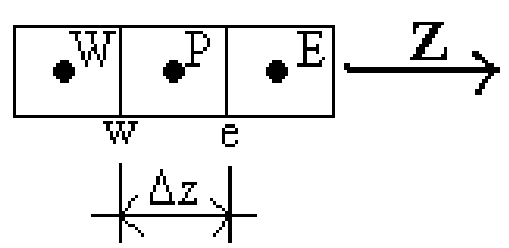

Figura 2: Domínio de integração unidimensional

2. As grandezas a, $L_{v}, C_{p g}, C_{p w}, C_{p a}, \rho_{g}$ e $\varepsilon$ são constantes $\forall z \in(0, L)$ e $t \in(0, \infty)$ e não são atualizados a cada iteração.

3. Os fluxos de massa $\left(\Phi_{m}\right)$ e calor $\left(\Phi_{h}\right)$ e a massa específica do ar $\left(\rho_{a}\right)$ são constantes para cada iteração temporal e espacial, mas são recalculados de uma iteração espacial para outra, com base nos valores de $\mathrm{X}, T_{g}$, Y e $T_{a}$ de outras iterações. Nas equações do grão, são calculados com valores das variáveis da iteração temporal anterior, da célula correspondente $\left(\Psi_{P}^{o}\right)$. Nas equações do ar, são calculados com valores das variáveis da iteração temporal presente e da célula anterior $\left(\Psi_{W}^{1}\right)$.

As integrações foram feitas considerando as seguintes hipóteses:

$$
\begin{aligned}
& \int_{t}^{t+\Delta t} \frac{\partial \Psi}{\partial t} d t=\Psi_{P}^{1}-\Psi_{P}^{o}, \\
& \int_{w}^{e} \Psi d z=* \Psi_{+}^{1} \triangle z, \\
& \int_{w}^{e} \frac{\partial \Psi}{\partial z} d z=\Psi_{e}^{1}-\Psi_{w}^{1}=\Psi_{P}^{1}-\Psi_{W}^{1},
\end{aligned}
$$

onde o subíndice indica a posição espacial, o superíndice indica a posição temporal e (+) é a posição espacial, que depende do esquema de interpolação escolhido.

\section{Equação de conservação da massa de água no grão}

Integrando a equação da umidade do grão em relação a t e a z e usando a hipótese (4.1) obtém-se a equação discretizada do teor de umidade do grão:

$$
X_{P}^{1}=-\frac{\Phi_{m} a}{\rho_{g}} \triangle t+X_{P}^{o}
$$

\section{Equação de conservação da energia no grão}

$\mathrm{Na}$ integração da equação da energia no grão, o teor de umidade do grão (X) é considerado constante e assume o valor atualizado da iteração temporal anterior. Usando a hipótese (4.1) obtém-se a equação de $T_{g}$ discretizada:

$$
T_{g P}^{1}=\frac{-a \triangle t}{\rho_{g}\left(C_{p g}+X_{P}^{o} C_{p w}\right)}\left(\Phi_{h}-\Phi_{m} L_{v}\right)+T_{g P}^{o} .
$$




\section{Equação de conservação da água no ar}

Integrando a equação (3.5) e usando a hipótese (4.3) obtém-se a equação discretizada do teor de umidade no ar.

$$
Y_{P}^{1}=-\frac{a(1-\varepsilon) \Phi_{m}}{V_{a} \rho_{a} \varepsilon} \triangle z+Y_{W}^{1} .
$$

\section{Equação de conservação da energia no ar}

As variáveis $T_{g}$ e $\mathrm{Y}$ são consideradas constantes na integração da equação da temperatura do ar e assumem o valor atualizado da presente iteração temporal. Usando as hipóteses (4.3) e (4.2) com $(+)=\mathrm{W}$, para integrar $T_{a}$ e $T_{g}$ no lado direito, obtém-se:

$$
T_{a P}^{1}=C \Phi_{m} C_{p v} \triangle z T_{g W}^{1}+\left(1-C \Phi_{m} C_{p v} \triangle z\right) T_{a w}^{1}+C \Phi_{m} \triangle z+C \Phi_{h} \triangle z .
$$

Usando as hipóteses (4.3) e (4.2) com $(+)=\mathrm{P}$, para integrar $T_{a}$ e $T_{g}$ no lado direito, obtém-se:

$$
\left(1+C \Phi_{m} C_{p v} \triangle z\right) T_{a P}^{1}=T_{a W}^{1}+C\left(\Phi_{m} C_{p v} T_{g P}^{1}+\Phi_{h}\right) \triangle z
$$

onde

$$
C=\frac{a(1-\varepsilon)}{V_{a} \varepsilon \rho_{a}\left(C_{p a}+C_{p v} Y_{p}^{1}\right)} .
$$

Usando as equações $(4.4 ; 4.5 ; 4.6 ; 4.7$ e 4.8$)$ pode-se determinar os coeficientes da equação geral do Método dos Volumes Finistos (MVF) na forma

\begin{tabular}{|c|c|c|c|c|}
\hline Var & $A_{P}$ & $A_{W}$ & $A_{E}$ & $\mathrm{~b}$ \\
\hline $\mathrm{X}$ & 1 & 0 & 0 & $-\frac{\Phi_{m} a}{\rho_{g}} \Delta t+X_{P}^{o}$ \\
\hline$T_{g}$ & 1 & 0 & 0 & $\frac{-a \triangle t\left(\Phi_{h}-\Phi_{m} L_{v}\right)}{\rho_{g}\left(C_{p g}+X_{P}^{o} C_{p w}\right)}+T_{g P}^{o}$ \\
\hline $\mathrm{Y}$ & 1 & 1 & 0 & $-\frac{a(1-\varepsilon) \Phi_{m}}{V_{a} \rho_{a} \varepsilon} \triangle z$ \\
\hline$T_{a}$ & 1 & $1-C \Phi_{m} C_{p v} \triangle z$ & 0 & $C\left(\Phi_{m} C_{p v} T_{g W}^{1}+\Phi_{h}\right) \triangle z$ \\
\hline$T_{a}$ & $1+C \Phi_{m} C_{p v} \triangle z$ & 1 & 0 & $C\left(\Phi_{m} C_{p v} T_{g P}^{1}+\Phi_{h}\right) \triangle z$ \\
\hline
\end{tabular}

$$
A_{P} \Psi_{P}=A_{W} \Psi_{W}+A_{E} \Psi_{E}+b,
$$

mostrados na Tabela 1, para os esquemas de interpolação utilizados.

Tabela 1 - Coeficientes da equação geral para volumes finitos 


\section{Resultados e Discussão}

Foram feitas simulações numéricas de secagem para as seguintes condições iniciais e de contorno: $\mathrm{X}=0,32 ; T_{g}=25^{\circ} \mathrm{C} ; \mathrm{Y}=0,0007 \mathrm{e} \mathrm{Ta}=25^{\circ} \mathrm{C}$ para $\mathrm{t}=0$ e $0 \leq z \leq L$ e $\mathrm{Y}=0,007$ e $T_{a}=50^{\circ} \mathrm{C}$ para $\mathrm{t}>0$ e $\mathrm{z}=0$.

As curvas obtidas mostram o comportamento próximo ao observado nos dados experimentais de Khatchatourian et al, [6].

\subsection{Comparação dos Resultados entre os Métodos de Difer- enças Finitas e Volume Finitos}

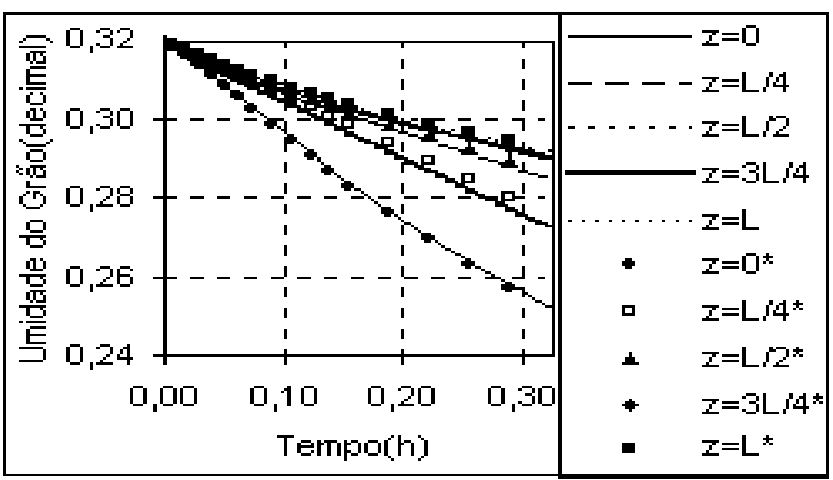

Figura 3: Comparação entre Diferenças Finitas $\left({ }^{*}\right)$ e Volumes Finitos: Teor de umidade do grão

Para ter uma referência de precisão, foi feita a comparação com os resultados de (Khatchatourian et al. [5]) usando o método de diferenças finitas, os quais apresentam boa concordância com resultados experimentais.

As Figuras 3 a 6 mostram a comparação dos resultados entre os dois métodos para o teor de umidade e temperatura do grão e do ar, em um intervalo de tempo de $\mathrm{t}=0,44 \mathrm{~h}$. Pequenas diferenças podem ser observadas nos resultados para $\mathrm{z}=\mathrm{L} / 4$ e provavelmente ocorreram devido ao posicionamento do centro (MVF) ou face (MDF) das células para tomada de dados. A boa coincidência entre as demais curvas permite concluir que os métodos produzem resultados muito semelhantes e podem ser usados independentemente.

Os tempos de execução computacional, para um PentiumII, $500 \mathrm{Mhz}$ e malhas de 2 a 90 células, com passo temporal de $3 \mathrm{~s}$ e tempo de secagem de $3 \mathrm{~h}$, foram relativamente pequenos: $2,8 \mathrm{~s}$ e $11 \mathrm{~s}$, respectivamente.

\subsection{Consideração de $T_{a P}$ e $T_{a W}$ na Equação da Temperatura do Ar}

A consideração de hipóteses diferentes para integrar $T_{a}$, cujo resultado é mostrado nas fórmulas (4.7) e (4.8) gera coeficientes diferentes na equação geral do MVF. 


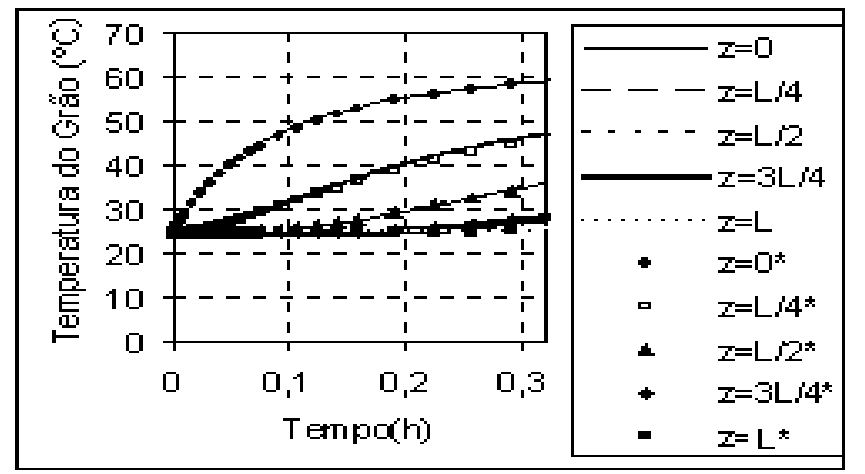

Figura 4: Comparação entre Diferenças Finitas $\left({ }^{*}\right)$ e Volumes Finitos: Temperatura do grão

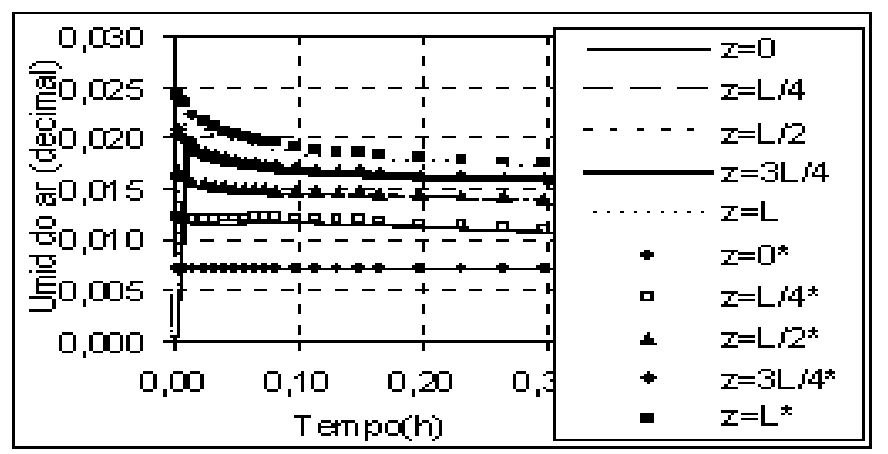

Figura 5: Comparação entre Diferenças Finitas $(*)$ e Volumes Finitos: Umidade do ar

Mesmo assim, os resultados são muito semelhantes, mesmo para malhas grandes (21 células). Sendo os métodos consistentes, quando $\triangle z \rightarrow 0$, os resultados tendem a aproximar-se. Os pontos $P_{1}$ e $P_{2}$ estão localizados em $\mathrm{z}=\mathrm{L} / 4$ e $\mathrm{z}=\mathrm{L}$ e foram plotados os valores da última iteração temporal.

\subsection{Escolha da Malha para Simulações Numéricas}

Para escolher uma malha adequada para execução confiável do programa, foram feitos testes variando o número de passos espaciais e temporais. Foram analisados os resultados das variáveis em vários pontos do domínio de integração, para diferentes malhas e $\Delta t=3 \mathrm{~s}$. Na medida que a malha diminui, a taxa de variação de cada variável tende a zero. Constatou-se que malhas com 21 passos espaciais podem ser usadas com relativa precisão. 


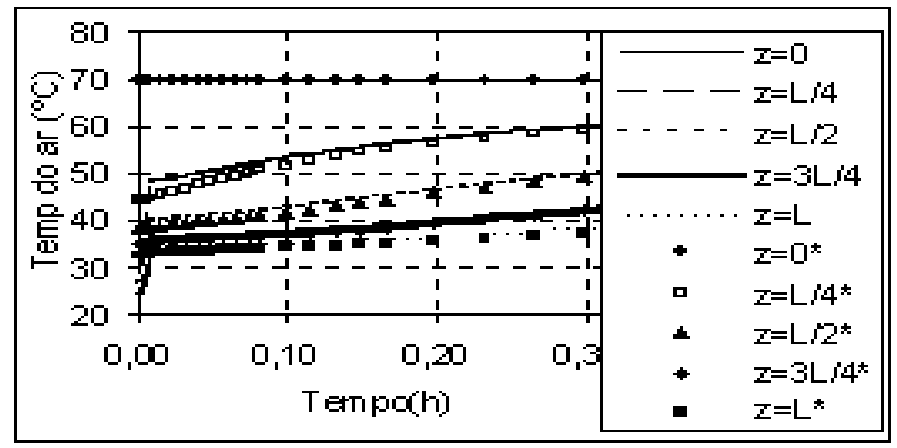

Figura 6: Comparação entre Diferenças Finitas $\left({ }^{*}\right)$ e Volumes Finitos: Temperatura do ar

\subsection{Convergência}

A convergência do método foi analisada analiticamente usando a hipótese da positividade dos coeficientes da equação geral do MVF (4.10) e $S_{p}$ negativo do termo fonte. Numericamente, a convergência foi analisada através de diversas execuções do programa com diferentes passos espaciais e temporais.

Nas equações de conservação da umidade do grão e do ar, a condição de convergência é satisfeita para qualquer $\Delta t$, pois o fluxo de massa é positivo.

$\mathrm{Na}$ equação de conservação da energia no grão, a condição de convergência só é satisfeita se $\left(\Phi_{h}+\Phi_{m} L v\right)<0$. Se os fluxos de calor e massa forem muito pequenos (em módulo) é possível que o acréscimo em $T_{g p} 0$ seja muito pequeno, para malhas espaciais grandes, negando a condição de convergência.

$\mathrm{Na}$ equação de conservação da energia no ar, a condição de convergência é dada por $\left|\Phi_{m} C_{p v} T_{g p}\right|<\left|\Phi_{h}\right|$. A ordem de grandeza dessas parcelas é muito próxima. Assim se o valor de $T_{g}$ crescer rápido, nas primeiras células e o fluxo de calor ficar próximo de zero, pode ocorrer negação da condição de convergência.

Como a convergência fica sujeita aos valores dos fluxos de massa e calor e estes aos valores da variáveis principais, pode-se concluir que existem situações de divergência, porém sua determinação analítica precisa é muito difícil.

Executando o programa para diferentes passos temporais, verificou-se que o maior passo de tempo possível é 3s. Para tempos maiores ocorre divergência nos valores de $T_{g}$ e $T_{a}$ (saem fora do domínio físico possível. Por exemplo, $T_{g P}>T_{g W}$ ou $T_{a}>T S$ ), nas primeiras iterações, confirmando análise feita acima. O método converge para um número mínimo de 4 passos espaciais, mas nesse caso os resultados não são muito precisos.

\section{Conclusão}

Foi desenvolvido um algoritmo computacional que resolve o sistema de equações diferenciais parciais para secagem de soja em leito fixo, usando o Método dos Volu- 
mes Finitos. A comparação entre os métodos de diferenças finitas e volumes finitos, mostrou que os dois métodos geram resultados praticamente iguais, com tempos computacionais ligeiramente diferentes, e portanto podem ser usados com sucesso em um aplicativo para controle de secadores.

Os testes mostraram que os resultados obtidos com $T_{a P}$ ou $T_{a W}$ na integração da equação da temperatura do ar são praticamente iguais em malhas com 21 ou mais volumes.

Para os regimes de secagem simulados o método dos volumes finitos é convergente e os passos temporal e espacial recomendados, considerando a precisão e o tempo de execução computacional para longos períodos de secagem, são 3s e 21 volumes, respectivamente.

\section{Lista de Símbolos}

a área superficial pela razão de volume do grão $\left(m^{2} / m^{3}\right)$

b.s. base seca

b.u. base úmida

$C_{p} \quad$ calor específico a pressão constante $(\mathrm{J} / \mathrm{kg} \mathrm{K})$

$L_{v} \quad$ calor latente de vaporização da água

$\mathrm{T}$ temperatura $\left({ }^{\circ} \mathrm{C}\right)$

TS temperatura de secagem $\left({ }^{\circ} \mathrm{C}\right)$

$V_{a} \quad$ velocidade do ar $(\mathrm{m} / \mathrm{s})$ na entrada da câmara de secagem

$\mathrm{X} \quad$ teor de umidade do grão em base úmida (\%)

$\mathrm{Y} \quad$ teor de umidade do ar em base seca $\left(k g_{w} / k g_{a}\right)$

z variável espacial (m)

$\rho \quad$ densidade específica ou massa específica $\left(\mathrm{kg} / \mathrm{m}^{3}\right)$

$\varepsilon \quad$ porosidade $\left(\mathrm{m}^{3} / \mathrm{m}^{3}\right)$

$\Psi \quad$ variável genérica

$\Phi_{m} \quad$ fluxo massa $\left(\mathrm{kg} / \mathrm{m}^{2} \mathrm{~s}\right)$

$\Phi_{h} \quad$ fluxo calor $\left(J / m^{4}\right)$

$\begin{array}{llll}a & \text { ar } & o & \text { tempo anterior } \\ g & \text { grão } & 1 & \text { tempo atual } \\ v & \text { vapor } & & \\ u & \text { úmido } & & \\ s & \text { seco } & & \\ w & \text { água } & \end{array}$




\section{Referências}

[1] F.W. Bakker-Arkema, W.G. Bickert e R.J. Patterson, Simultaneous heat and mass transfer during the cooling of a deep bed of biological products under varying inlet air conditions, (1967), 297-307.

[2] D.B. Brooker, F.W. Bakker-Arkema e C.W. Hall, "Drying Cereal Grains", The Avi Publishing Company, Westport, 1974.

[3] F. Courtois, A. Lebert, J.C. Lasseran e J.J. Bimbenet, Simulation of industrial dryers: solving numerical and computer problems, 927-945.

(1991)

[4] A.S. França, "Simulação Bidimensional de Secadores de Grãos Cereais", Dissertação de Mestrado, Departamento de Engenharia Mecânica, UFMG, MG, Brasil, 1991.

[5] O.A. Khatchatourian, N.A. Toniazzo e P.A.P. Borges, Um modelo simples para análise do processo de secagem de soja em camada móvel com fluxo contrário de ar, em "Cilamce/99", São Paulo, 1999.

[6] O.A. Khatchatourian, P.A.P. Borges e V.A. Petry, Modelo matemático e análise comparativa de métodos numéricos para simulação do processo de secagem de soja em câmara de leito fixo, em "XXIII CNMAC", Santos, SP, 2000.

[7] C.R. Maliska, "Transferência do Calor e Mecânica dos Fluidos Computacional", LTC, Rio de Janeiro, 1985.

[8] S.V. Patankar, "Numerical Heat Transfer and Fluid Flow", McGraw Hill, New York, 1980. 